\title{
Role of Fine Needle Aspiration Cytology in Evaluation of Cervical Lymphadenopathy
}

\author{
*Dr. Veenita Singh, Dr. Mohit Srivastava, Dr. Rajesh Roshan, \\ Dr. Deepak Khanna, Dr. Harsimrat Dhingra \\ *Corresponding author: Dr. Veenita Singh *
}

\begin{abstract}
Introduction: Cervical lymphadenopathy is one of the commonest clinical presentations of patients of head and neck pathologies. Metastatic cervical lymphadenopathy is a common presentation in head and neck oncology. The aim of this study is to evaluate the effectiveness of FNAC as a primary investigation tool.

Aims and Objectives: The aim of the study was to evaluate the effectiveness of FNAC in diagnosing cervical lymphadenopathy.

Material and Methods: A study was conducted in the Department of Dentistry and E.N.T., SIMS, Hapur, U.P., India from January 2016 to February 2017 which included 52 patients with cervical lymphadenopathy presenting to outpatient clinics. FNAC was done in patients of cervical lymphadenopathy before surgery or incision/excision biopsy and the results of FNAC were correlated with histopathological evaluation.

Results: In our study 88.47.\% of sensitivity and specificity of $92.03 \%$ was found on FNAC of cervical lymph nodes.

Conclusion: FNAC remains a safe, cost effective and appropriate first line investigation though the results have to be confirmed with HPE for proper evaluation and management.
\end{abstract}

Keywords: Fine Needle Aspiration Cytology, Cervical Lymphadenopathy

\section{Introduction}

Most head and neck tumors may present to the clinician as neck nodes. Depending on the primary site, up to $80 \%$ of patients with upper aero digestive mucosal malignancy will have cervical nodal metastasis at presentation. The occurrence of nodal metastasis has a profound effect on the management and prognosis of these patients. Cervical Lymphadenopathy is one of the commonest clinical presentations of patients, attending the outdoor clinics in most hospitals. The a etiology varies from an inflammatory process to a malignant condition1.Fine needle aspiration cytology is a simple, quick and inexpensive method that is used to sample superficial masses like those found in the neck and is usually performed in the outpatient clinic. It causes minimal trauma to the patient and carries virtually no risk of complications.

Masses located within the region of the head and neck can be readily diagnosed using this technique2, 3. FNAC is both diagnostic and therapeutic in a cystic swelling 4. It is an initial and well established technique for the management of patients presenting with head and neck lumps. 5-8 FNAC is helpful in differentiating between a malignant and a benign tumour with over $90 \%$ accuracy9. It is helpful in the work-up of cervical masses and nodules because biopsy of cervical adenopathy should be avoided unless all other diagnostic modalities have failed to establish a diagnosis10. FNAC does not give the same architectural detail as histology but it can

Provide cells from the entire lesion as many passes through the lesion can be made while aspirating 11. Limitation of FNAC lies in the fact that it does not allow evaluation of the morphology of the lesion. Falsenegative and false-positive results are known to occur in FNAC Due to which a definitive diagnosis is necessary for cases with clinical suspicion. The purpose of our study was to ascertain the reliability of FNAC in the diagnosis of cervical lymphadenopathy.

\section{Materials And Method}

This is a prospective study carried out at Department of Dentistry and Otolaryngology, Saraswathi Institute of Medical Sciences, Hapur, Ghaziabad, Uttar Pradesh, from January 2016 to February 2017 which included 52 patients with cervical lymphadenopathy presenting to the outpatient clinics. FNAC procedures were done in all the patients before surgery after a thorough physical examination. Palpable neck masses were aspirated using $23 \mathrm{G}$ needle and $20 \mathrm{ml}$ syringe. A negative pressure was applied to the syringe by pulling the syringe plunger and at least two dry specimens and two alcohol fixed specimens were taken. The alcohol fixed smears were immediately submerged in $95 \%$ ethyl alcohol. All the slides preparations were made by the trained staff of Pathology Department. Alcohol fixed smears were subsequently stained with Haemotoxylin and Eosin 
stains. The results of the FNAC were evaluated into inflammatory process, tubercular and malignant disorder. Comparisons were made when patients were subjected to surgery and biopsies were sent to the Department of Pathology for evaluating the mass. After correlating the specimens with available histological findings obtained by surgery and from the patient's records, accuracy, sensitivity, specificity were calculated.

\section{Results}

The study included 52 patients with cervical lymphadenopathies. There were 32 female and 20 male patients with an age range of 14-68 years. Out of 52 patients who underwent FNAC for cervical lymhadenopathy $34(65.3 \%)$ came out to be inflammatory, $10(19.2 \%)$ tubercular, $6(11.5 \%)$ malignancy, and 2 (3.8\%) came out inconclusive or as blood aspirate.

All the patients who underwent FNAC in the study group were subjected to excision/incision biopsy to confirm then diagnosis. Out of 34 inflammatory nodes reported in FNAC, 8 came out to be other lesions on HPE. The sensitivity for inflammatory nodes was found to be $90.9 \%$ while specificity was $84.61 \%$. Sensitivity for tubercular lymphadenitis was found to be $88.8 \%$ and specificity $95.65 \%$, and for malignant nodes sensitivity was $85.71 \%$ and sensitivity $95.83 \%$. The overall sensitivity was $88.47 \%$ and specificity was $92.03 \%$.

\section{Discussion}

Cervical lympadenopathy often pose a challenging diagnostic problem to the clinician. Surgical biopsy is the commonest method of tissue diagnosis, FNAC is in practice since the 1930s. This method has become popular as a diagnostic step in the evaluation of a head and neck mass. 12 In our study the cases of FNAC revealed $88.47 \%$ of sensitivity and specificity of $92.03 \%$. less than $100 \%$ sensitivity of the cervical lymphadenopathies demonstrates the fact that we cannot always rule out disease by depending exclusively on FNAC. Sensitivity for tubercular lymphadenitis was found to be $88.8 \%$ and specificity $95.65 \%$, and for malignant nodes sensitivity was $85.71 \%$ and sensitivity $95.83 \%$. According to a study the diagnostic accuracy of FNAC in metastatic disease varies from $87 \%$ to $97.9 \% 13,14$ and for lymphomas is $82 \% 13$. The sensitivity of FNAC for metastatic lesions to lymph nodes has varied from $97.9 \%$ to $100 \%$, whereas the specificity has been found to be $100 \% .13,15$ For the lymphomas the sensitivity has been found to be $80 \%$ and specificity $100 \% 15$ Although the majority of procedures were performed by the pathologists, there were no statistical differences in the results of FNACs performed by an attending or by residents in the second half of their residency. In other words, the learning period does not require years of experience and procedure is easy to master. Moreover, it can be performed in outpatient clinics, as was done in our study. In about $75 \%$ of patients, a single FNAC was needed for evaluation and no further aspirate was required making this tool highly efficient for diagnosing masses in the head and neck region. FNAC is only one step in the overall evaluation of patients with cervical lympadenopathy, and one should not rely exclusively on FNAC results. Negative FNACs results in patients with a high index of suspicion for malignancy were further investigated by removing the suspicious mass surgically. FNAC is a safer choice for initial evaluation of masses suspicious of malignancy as the frequency of needle seeding of tumor cells in the procedure is reported as low as 0.003-0.009\%. 16 Hence, considering this range, tumor risk in FNAC is almost nonexistent in comparison to excision biopsy. To avoid or minimize false positive results various factors including regenerative changes, metaplasia and others should be taken into considerations while reporting. False negative results may be due to cystic change, necrotic and hemorrhagic areas revealing no diagnostic cellular yield.17 With increasing cost of medical facilities, any technique which speeds up the process of diagnosis, limits the physical and psychological trauma to the patient, and saves the expenditure of hospitalization, will be of Tremendous value.18 It was concluded that FNAC is an easy and suitable investigation tool for the primary assessment of patients with cervical lymphadenopathy on OPD basis. Its diagnostic accuracy is variable.

\section{Acknowledgements}

The authors declare there is no conflict of interest. No source of funding for the study. Ethical approval was taken prior to the study from the ethical committee of the Saraswathi Institute of Medical Sciences.

\section{References}

[1]. Hirachand S, Lakhey M, Akhter J, Thapa B. Evaluation of fine needle aspiration cytology of lymph nodes in Kathmandu Medical College, Teaching hospital. Kathmandu Univ Med J 2009;7(26):139-42

[2]. Chauhan Setal, Dharmendra Rathod, D.S. Joshi. FNAC of Swellings of Head and Neck Region. Indian Journal of Applied Basic Medical Sciences, 2011;13:1-6

[3]. Gamba PG, Messineo A, Antoniello LM, Boccato P, Blandamura S, Cecchetto G, et al. A simple exam to screen superficial masses, FNAC. Med Pediatr Oncol.,1995;24:97-9

[4]. Afridi S, Malik K, Waheed I. Role of fine needle aspiration biopsy and cytology in breast lumps. J Coll Physicians Surg Pak, 1995; 5:75-7.

[5]. Anne S, Teot LA, Mandell DL. Fine needle aspiration biopsy: role in diagnosis of paediatric head and neck masses. Int J Pediatr Otolaryngol 2008;72(10):1547-53 
[6]. Bajaj Y, De M, Thompson A. Fine needle aspiration cytology in diagnosis and management of thyroid disease. J Laryngol Otol 2006;120(6): 467-9

[7]. Murthy P, Laing MR, Palmer TJ. Fine needle aspiration cytology of head and neck lesions: an early experience. J R Coll Surg Edinb 1997; 42(5): 341-6.

[8]. Sack MJ, Weber RS, Weinstein GS, Chalian AA, Nisenbaum HL, Yousem DM. Image-guided fine-needle aspiration of the head and neck: five years experience. Arch Otolaryngol Head Neck Surg 1998; 124(10): 1155-61.

[9]. Burnand KG, Young AE, Lucas J, Rrolands BJ, Scholefield J. The new Aird's companion in surgical studies. 3 rd edition, China: Elsevier; 2005.

[10]. Layfield LJ. Fine-needle aspiration of the head and neck. Pathology (Phila), 1996;4:409-38.

[11]. Kirk RM, Ribbans WJ. Clinical Surgery in General. 4th edition, Edinburgh: Elsevier; 2004.

[12]. Raju G, Kakar PK, Das DK, Dhingra PL, Bhambhani S. Role of fine needle aspiration biopsy in head and neck tumours. The J of Laryngol and Otol 1988; 102:248-51.

[13]. Alam K, Khan A, Siddiqui F, Jain A, Haider N, Maheshwari V. Fine needle aspiration cytology (FNAC): A handy tool for metastatic lymphadenopathy. Int J Pathol. 2010;10:2.

[14]. Khajuria R, Goswami KC, Singh K, Dubey VK. Pattern of lymphadenopathy on fine needle aspiration cytology in Jammu. JK Sci. 2006;8:157-9.

[15]. Hirachand S, Lakhey M, Akhter P, Thapa B. Evaluation of fine needle aspiration cytology of lymph nodes in Kathmandu Medical College, Teaching hospital. Kathmandu Univ Med J. 2009;7:139-42.

[16]. Mohamed MH, Hitam S, Brito-Mutunayagam S, Yunus MRM. Role of FNAC in evaluation of of neck masses. J Curr Surg. 2013;3(1):19- 23

[17]. Suryawanshi Kishor H.et.al Spectrum of fnac in palpable head and neck lesions in a tertiary care hospital in India- a 3 years study; Indian Journal of Pathology and Oncology, January - March 2015; 2(1); 7-13.

[18]. Rathod GB, Parmar P. Fine needle aspiration cytology of swellings of head and neck region. Indian J Med Sci 2012(cited 2015 Oct 12); 66:49-54. 